\title{
Closure of multiple ventricular septal defects by the felt sandwich technique: Further analysis of 36 patients
}

\author{
Hirohisa Murakami, MD, ${ }^{\mathrm{b}}$ Naoki Yoshimura, MD, ${ }^{\mathrm{b}}$ Hiroaki Takahashi, MD, ${ }^{a}$ Hironori Matsuhisa, MD, ${ }^{a}$ \\ Masahiro Yoshida, MD, ${ }^{a}$ Yoshihiro Oshima, MD, ${ }^{a}$ Takuro Misaki, MD, ${ }^{b}$ and Masahiro Yamaguchi, MDa
}

Objectives: We previously described a novel technique for closure of trabecular ventricular septal defects by sandwiching the septum with 2 polyester felt patches without requiring left ventriculotomy. We describe the midterm results of this technique and the postoperative cardiac function.

Methods: This is a retrospective study of 36 consecutive patients. The median age and body weight at the time of repair was 15 months (range: 2-115 months) and $7.8 \mathrm{~kg}$ (range: $3.9-51.9 \mathrm{~kg}$ ), respectively.

Results: Sixty-three trabecular ventricular septal defects in 36 patients were closed with the felt sandwich technique. In the early postoperative period, 1 patient died of pulmonary hypertensive crisis. There were 2 late deaths. One patient died of pneumonia 6 months after surgery, and another died suddenly of ventricular arrhythmias 2 years after surgery. Three patients required reoperation (closure of major residual ventricular septal defect, cardiac transplantation for dilated cardiomyopathy, and pacemaker implantation for complete atrioventricular block). Postoperative left ventricular fractional shortening in the group with a body surface area less than $0.4 \mathrm{~m}^{2}$ was significantly lower than that in the group with a body surface area of $0.4 \mathrm{~m}^{2}$ or greater $(0.22 \pm 0.09$ vs $0.31 \pm 0.06, P=.0027)$. Moreover, there was a strong correlation between postoperative left ventricular ejection fraction and total patch area/body surface area ratio $(\mathrm{R}=-0.74, P=.0004)$.

Conclusion: Multiple trabecular ventricular septal defects can be closed with the felt sandwich technique easily and safely. Although this technique can be used in small infants, the use of numerous felt patches disturbs the movement of ventricular septum, which may cause postoperative cardiac dysfunction.

From the Department of Cardiothoracic Surgery, Kobe Children's Hospital, ${ }^{\mathrm{a}}$ Kobe, Japan, and Department of Surgery, Division of I, Toyama University, School of Medicine,' Toyama, Japan.

Received for publication Feb 16, 2006; accepted for publication April 18, 2006.

Address for reprints: Hirohisa Murakami, MD, Department of Surgery, Division of I, Toyama University, School of Medicine, 2630 Sugitani, Toyama 930-0194, Japan (E-mail: murahisa@ms.toyama-mpu.ac.jp).

J Thorac Cardiovasc Surg 2006;132:278-82

$0022-5223 / \$ 32.00$

Copyright $\odot 2006$ by The American Association for Thoracic Surgery

doi:10.1016/j.jtcvs.2006.04.008
$\mathrm{T}$ he repair of multiple trabecular ventricular septal defects (VSDs) remains controversial and a surgical challenge. These VSDs are difficult to localize precisely, and the omission of the VSDs during repair may lead to a postoperative residual shunt. It is well known that ventriculotomy for closure of VSDs sometimes causes postoperative ventricular dysfunction or arrhythmia. Although improved techniques for the repairs of such VSDs without a ventriculotomy have been reported, ${ }^{1}$ morbidity and mortality remain high because of the presence of residual VSDs and postoperative myocardial dysfunction.

We previously described a novel, safe, and simple technique for closure of trabecular VSDs by sandwiching the septum between 2 polyester felt patches placed in the left and right ventricle without ventriculotomy (the felt sandwich technique). ${ }^{2,3}$ However, midterm or long-term outcomes of patients undergoing this technique have not been reported. Moreover, the influence of numerous patches placed in the interventricular septum on cardiac function is unclear.

In this study, we investigated the midterm results of this technique and performed further analysis of postoperative cardiac function to determine the appropriate indications and operative method for this type of procedure. 


\section{Abbreviations and Acronyms \\ ACEI $=$ angiotensin-converting enzyme inhibitor \\ $\mathrm{BSA}=$ body surface area \\ $\mathrm{PAB}=$ pulmonary artery banding \\ VSD $=$ ventricular septal defect}

\section{Patients and Methods \\ Patients}

From November 1998 to August 2005, 36 consecutive patients underwent surgical treatment for multiple trabecular VSDs with the felt sandwich technique at our institutes. Patients or guardians of the patients gave informed preoperative consent. There were 21 boys and 15 girls. The patients ranged in age from 2 to 115 months (median: 15 months) and weighed 3.9 to $51.9 \mathrm{~kg}$ (median: $7.8 \mathrm{~kg}$ ). Associated cardiac anomalies included tetralogy of Fallot in 4 patients, double outlet right ventricle in 3 patients, atrioventricular septal defect in 2 patients, transposition of the great arteries with coarctation of aorta in 1 patient, and atrial septal defect patients in 9 patients. Eleven patients had isolated multiple VSDs. Multiple VSDs were diagnosed preoperatively in 25 patients $(69 \%)$, and trabecular VSDs were identified intraoperatively in 11 patients (31\%). Pulmonary artery banding (PAB) was previously performed in 13 patients (Table 1).

\section{Felt Sandwich Technique for Closure of Trabecular Ventricular Septal Defects}

The detail of the surgical technique was described in previous reports. ${ }^{2,3}$ Briefly, under moderate hypothermic total cardiopulmonary bypass, the aorta was crossclamped, and myocardial protection was achieved with cold-blood antegrade cardioplegia. A longitudinal right atriotomy was performed, and the trabecular septum was exposed through the tricuspid valve. We placed a right-angled forceps between the right-sided trabecular VSD through the tricuspid valve and the left-sided trabecular VSD through another perimembranous VSD or a longitudinal atrioseptostomy. Through the trabecular VSD, the forceps were used to advance a $3 F$ Nelaton catheter (Bard, Haverhill, Mass), which was used to place an oversized circular polyester felt patch mounted on a 3-0 Nespolen suture (Azwell, Osaka, Japan). The suture ends were passed through a slightly smaller polyester felt patch on the right ventricular side of the septum. The Nespolen suture was then tied, thereby sandwiching the septum between 2 polyester felt patches. Early in this series, the felt size used on the left ventricular side was 8 to $10 \mathrm{~mm}$ larger than the estimated size of the VSD. Recently, a slightly smaller felt ( 6 to $8 \mathrm{~mm}$ larger than the estimated VSD size) was used to avoid ventricular dysfunction by felt patches placed in the interventricular septum.

\section{Postoperative Cardiac Function}

Cardiac function was evaluated by echocardiography and cardiac catheterization 4 weeks after the operation because patches occupying the interventricular septal space may cause left ventricular dysfunction. The patients were divided into 2 groups based on a body surface area (BSA) less than $0.4 \mathrm{~m}^{2}$ or greater than $0.4 \mathrm{~m}^{2}$, and their cardiac function was compared. We evaluated the correlation between left ventricular ejection fraction and the value of the calculated total patch area/BSA ratio based on the following equation: total patch area/BSA $=$ the sum total patch area of patches used for closure of trabecular and perimembranous or other VSDs $\left(\mathrm{cm}^{2}\right) / \mathrm{BSA}\left(\mathrm{m}^{2}\right)$.

\section{Statistics}

Data for continuous variables are expressed as the median value and range. Measured valuables are expressed as the mean \pm standard deviation. The Student $t$ test was used to compare echocardiographic fractional shortening and ejection fraction during catheterization.

\section{Results}

\section{Early Results}

One patient with 21-trisomy died of a pulmonary hypertensive crisis 1 day after the operation. One patient had a complete atrioventricular block and required pacemaker implantation.

\section{Midterm Results}

There were 2 late deaths. One patient with double outlet right ventricle died suddenly of ventricular arrhythmia 2 years after the operation. Another patient with tetralogy of Fallot and bronchomalacia died of pneumonia 6 months after the operation. Two patients required reoperation. One patient with transposition of the great arteries underwent an arterial switch operation and closure of the VSDs at the age of 2 months and at a weight of $4.5 \mathrm{~kg}$. At that time, multiple trabecular VSDs were identified and 4 VSDs in total were closed. Severe left ventricular dysfunction developed postoperatively. She required a heart transplantation 18 months after the operation.

Another patient had postoperative congestive heart failure after closure of 2 VSDs. Echocardiography and cardiac catheterization revealed a large residual VSD with a pulmonary blood flow/systemic blood flow (Qp/ Qs) of 1.73. At the time of reoperation, a muscular inlettype VSD was revealed, which was closed with a standard patch closure. Operative findings demonstrated that the felt patch from the previous operation was covered with proliferating endocardium.

\section{Closure of Trabecular Ventricular Septal Defects}

Sixty-three trabecular VSDs were closed in 36 patients with the felt sandwich technique. The VSD included 7 high trabecular, 29 mid-trabecular, and 27 low trabecular septal lesions according to the classification of Serraf and

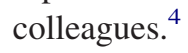

Major leakage around the felt patch was found in 1 patient, although the leakage disappeared spontaneously 14 months later. Minor leakage was found perioperatively in 11 patients. During follow-up, minor leakage disappeared in 8 of these 11 patients. 
TABLE 1. Characteristics of 36 patients undergoing felt patch closure for trabecular ventricular septal defects

\begin{tabular}{|c|c|c|c|c|c|}
\hline Case & Associated cardiac anomaly & Number of VSDs & Previous intervention & Preoperative Dx of tVSD & BW (kg) \\
\hline 1 & DORV, PA & $2(1)$ & Bilateral BT & Pre. & 51.9 \\
\hline 2 & VSD & $3(2)$ & PAB & Pre. & 18.3 \\
\hline 3 & DORV, PS & $2(1)$ & Bilateral BT & Pre. & 18 \\
\hline 4 & AVSD, TF & $2(1)$ & Right BT & Pre. & 12.9 \\
\hline 5 & ASD & $2(1)$ & - & Intraop. & 4.6 \\
\hline 6 & MR & $2(1)$ & - & Intraop. & 7.8 \\
\hline 7 & VSD & $3(2)$ & PAB & Pre. & 18.2 \\
\hline 8 & PS & $2(1)$ & - & Pre. & 14.1 \\
\hline 9 & VSD & $4(3)$ & PAB & Pre. & 16.5 \\
\hline 10 & ASD, PDA & $3(2)$ & PAB & Pre. & 14.1 \\
\hline 11 & VSD & $2(1)$ & - & Pre. & 6.5 \\
\hline 12 & ASD & $3(2)$ & PAB & Pre. & 5.9 \\
\hline 13 & ASD & $3(2)$ & PAB & Pre. & 9.6 \\
\hline 14 & ASD & $2(1)$ & - & Pre. & 7.4 \\
\hline 15 & ASD & $3(2)$ & - & Pre. & 4.1 \\
\hline 16 & VSD & $2(1)$ & PAB & Pre. & 10.4 \\
\hline 17 & ASD & $2(1)$ & - & Intraop. & 4.5 \\
\hline 18 & DORV, PS & $3(2)$ & - & Pre. & 6.6 \\
\hline 19 & VSD & $2(1)$ & PAB & Pre. & 11.6 \\
\hline 20 & $\mathrm{TGA}(\mathrm{II}), \mathrm{CoA}$ & $4(3)$ & $\mathrm{EAAA}+\mathrm{PAB}$ & Intraop. & 4.5 \\
\hline 21 & ASD & $2(1)$ & - & Pre. & 3.9 \\
\hline 22 & IVC interruption & $4(3)$ & PAB & Pre. & 7.8 \\
\hline 23 & ASD, PAPVR & $4(3)$ & $\mathrm{PAB}$ & Pre. & 7.1 \\
\hline 24 & AVSD, CoA & $3(2)$ & $\mathrm{EAAA}+\mathrm{PAB}$ & Pre. & 8.4 \\
\hline 25 & DAA, PDA & $4(3)$ & Left arch division + PAB & Pre. & 10.3 \\
\hline 26 & VSD & $3(2)$ & - & Pre. & 5.6 \\
\hline 27 & TS & $5(4)$ & - & Intraop. & 6.6 \\
\hline 28 & TF, PAPVR & $3(2)$ & - & Pre. & 5.23 \\
\hline 29 & VSD & $4(3)$ & - & Intraop. & 6.6 \\
\hline 30 & VSD & $2(1)$ & - & Intraop. & 6.9 \\
\hline 31 & MR & $4(3)$ & - & Pre. & 6.7 \\
\hline 32 & $\mathrm{TF}, \mathrm{ASD}$ & $3(2)$ & - & Pre. & 11.3 \\
\hline 33 & TF, ASD & $2(1)$ & - & Intraop. & 10.1 \\
\hline 34 & $\mathrm{TF}$ & $2(1)$ & - & Intraop. & 9.4 \\
\hline 35 & VSD & $2(1)$ & - & Intraop. & 3.9 \\
\hline 36 & VSD & $2(1)$ & - & Intraop. & 5.8 \\
\hline
\end{tabular}

The numbers of trabecular VSDs are shown in parentheses. $D x$, Diagnosis; $t V S D$, trabecular VSD; DORV, double-outlet right ventricle; $P A$, pulmonary atresia; $P S$, pulmonary stenosis; $A V S D$, atrioventricular septal defect; TF, tetralogy of Fallot; $A S D$, atrial septal defect; $M R$, mitral regurgitation; $P D A$, patent ductus arteriosus; TGA, transposition of the great arteries; IVC, inferior vena cava; PAPVR, partial anomalous pulmonary venous return; CoA, coarctation of the aorta; $D A A$, double aortic arch; $T S$, tricuspid valve stenosis. $B T$, Blalock-Taussig shunt; $P A B$, pulmonary artery banding; $E A A A$, extended aortic arch anastomosis; intraop., intraoperative identification.

The mean size of the trabecular VSD was $4.1 \pm 1.2 \mathrm{~mm}$, and the mean sizes of the left- and right-sided patches were $10.9 \pm 2.8 \mathrm{~mm}$ and $8.9 \pm 2.6 \mathrm{~mm}$, respectively. In the most recent 19 patients, left- and right-sided patches were $8.6 \pm$ $1.4 \mathrm{~mm}$ and $6.6 \pm 1.3 \mathrm{~mm}$, respectively, for $3.5 \pm 0.7 \mathrm{~mm}$ trabecular VSDs. In the 17 patients undergoing the procedure in the earlier era, left- and right-sided patches were $12.8 \pm 2.3 \mathrm{~mm}$ and $10.8 \pm 1.9 \mathrm{~mm}$, respectively, for a mean of VSD size of $4.5 \pm 1.7 \mathrm{~mm}$. The sizes of felt patches were significantly smaller in the current cases than those in the early cases (Figure 1). There was no difference in the ratio of leakage around the felt patch between early and current cases.

\section{Cardiac Function}

Cardiac function was evaluated by echocardiography (34 patients) and cardiac catheterization (21 patients). Left ventricular fractional shortening in the group with a BSA less than $0.4 \mathrm{~m}^{2}(\mathrm{n}=15)$ was significantly lower than that in the group with a BSA greater than $0.4 \mathrm{~m}^{2}(\mathrm{n}=18)$ $(0.22 \pm 0.09$ vs $0.31 \pm 0.06, P=.007)$ (Figure 2$)$. Left ventricular ejection fraction was strongly correlated with 


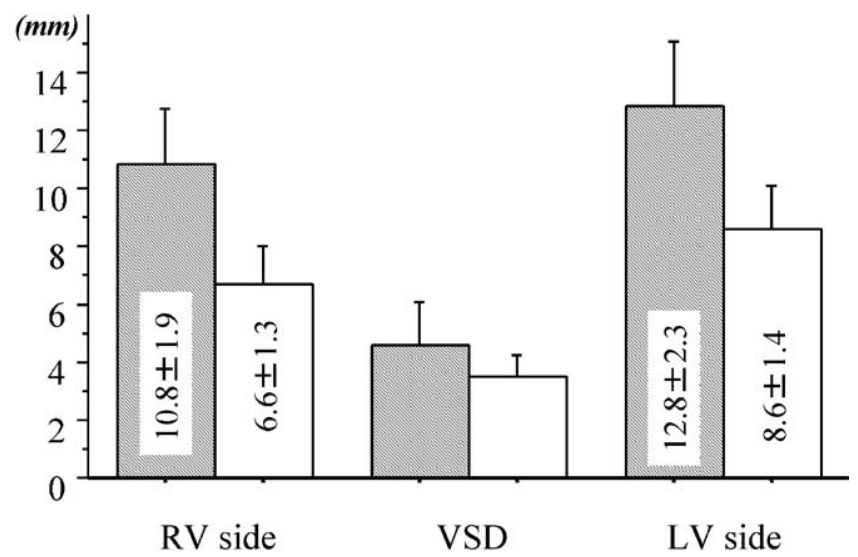

Figure 1. Relationship between the size of the trabecular VSDs and the felt patch. The sizes of trabecular VSD and felt patch are shown for early cases (hatched bar, 18 patients) and recent cases (open bar, 17 patients). The size of felt patches in the recent cases was significantly smaller than those in the early cases. VSD, Ventricular septal defect; $R V$, right ventricle; $L V$, left ventricle.

total patch area/BSA ratio $(\mathrm{R}=-0.74, P=.0004)$ (Figure 3).

\section{Discussion}

Left ventriculotomy for closure of multiple VSDs leads to left ventricular dysfunction, aneurysm formation, and ventricular arrhythmias in both the early and late postoperative periods. ${ }^{5}$ To avoid these complications, several surgical maneuvers to treat multiple VSDs have been described. ${ }^{1,6}$

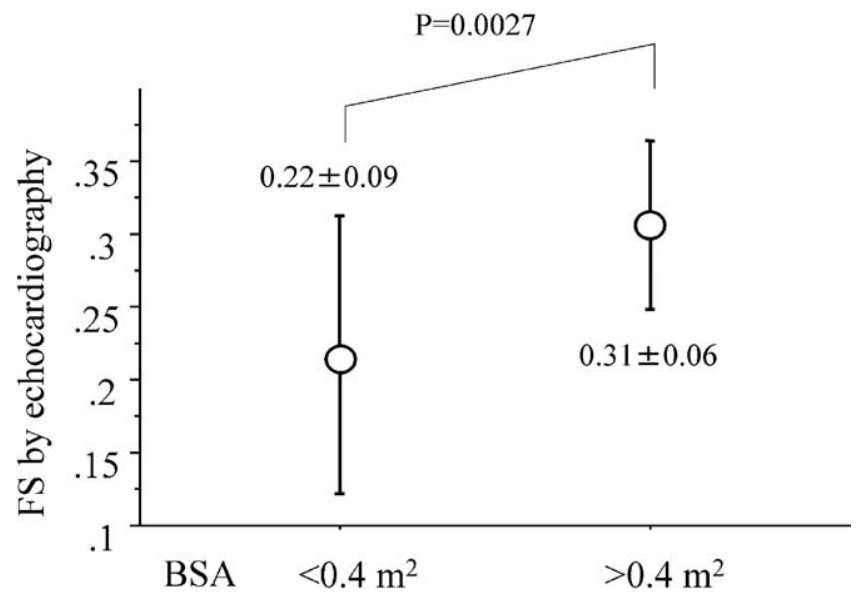

Figure 2. Comparison of postoperative cardiac function between patients with a BSA less than $0.4 \mathrm{~m}^{2}$ and greater than $0.4 \mathrm{~m}^{2}$. Left ventricular fractional shortening in the group with a BSA less than $0.4 \mathrm{~m}^{2}$ ( $\mathrm{n}=14$ ) was significantly lower than that in the group with a BSA greater than $0.4 \mathrm{~m}^{2}(\mathrm{n}=18)(0.22 \pm 0.09$ vs $0.31 \pm 0.06$, $P=.0027)$. BSA, Body surface area; FS, fraction shortening.
Transcatheter devices for percutaneous or surgical closure of muscular VSD have been frequently reported. ${ }^{7-9}$ However, the mortality and morbidity associated with these procedures were still high. ${ }^{9,10}$ Furthermore, postoperative cardiac function and long-term outcomes were unclear.

Kitagawa and colleagues ${ }^{1}$ described the technique of VSD repair with transection of the moderator band, without left ventriculotomy. Left ventricular function after this procedure, particularly ventricular septal movement, was not described in long-term follow-up. However, patients required prolonged medication for cardiac failure, including diuretics and angiotensin-converting enzyme inhibitor (ACEI). This suggests that this procedure is associated with impaired cardiac function postoperatively. In our cases, 20 of 33 survivors received no drug postoperatively. In the other 13 patients, 5 patients required ACEI, 5 patients required both $\mathrm{ACEI}$ and diuretics, and 3 patients required ACEI, diuretics, and digitalis. Eight patients who were administered the drug had a BSA less than $0.4 \mathrm{~m}^{2}$ at the time of operation. Macé and colleagues ${ }^{6}$ described the technique of repair for multiple VSDs using a whole ventricular septal patch. Early postoperative cardiac function was good. However, it was hypothesized that the whole patch may disturb growth of the left and right ventricles, impairing cardiac function.

In 2001, we described the felt sandwich technique for closure of trabecular VSDs. This simple technique does not require special devices, prolonged operation time, or left ventriculotomy. This technique enabled us to close trabecular VSDs easily, which were identified intraoperatively. Brizard and colleagues ${ }^{11}$ reported a similar technique using intraoperative echocardiography guidance with excellent results.

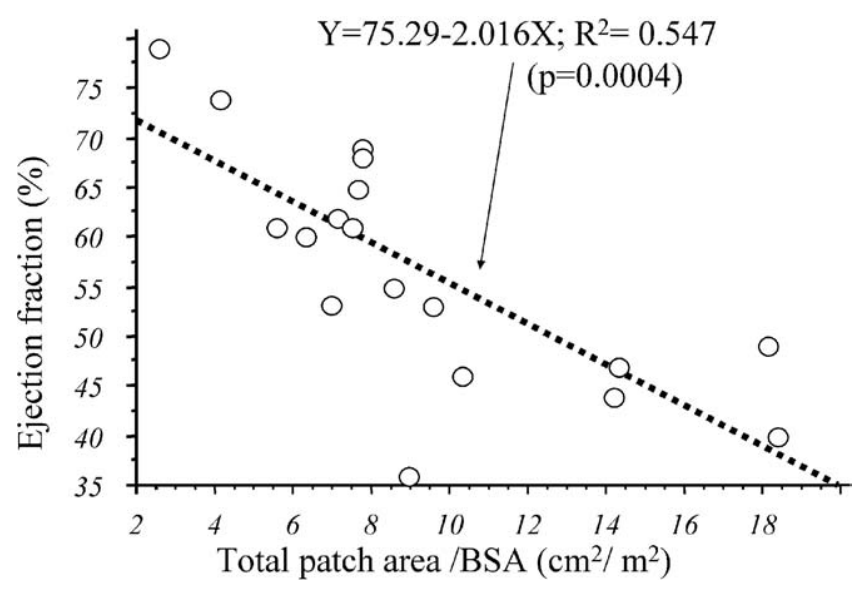

Figure 3. Relationship between ejection fraction based on cardiac catheterization and total patch area/BSA ratio. Raw data (circles). The regression curve (broken line) shows a linear decrease with a significant correlation as total patch area/BSA ratio increases $(R=-0.74, P=.0004)$. $B S A$, Body surface area. 
Although the felt sandwich technique is simple, safe, effective, and reproducible, this technique was found to be associated with leakage around the felt patch and left ventricular dysfunction because the use of numerous patches disturbs the movement of the ventricular septum. We identified some infants in whom deterioration of cardiac function developed. Therefore, we performed further analyses to assess midterm results, fate of the residual shunt, and postoperative cardiac function.

In our series, there was major perioperatively leakage around the felt patch in 1 patient and minor leakage in 11 patients. During follow-up, we observed spontaneous closure of a major leakage 14 months after the operation. Minor leakages in 8 of 11 patients disappeared. Although minor leakage in 3 patients persisted, the leakage is expected to disappear in the near future. These results indicated that the residual shunt around the felt patch disappears spontaneously in most cases. However, overlooked trabecular VSDs never disappeared. We identified 1 patient who required reoperation because of a large residual VSD.

Preoperative detection of the location and number of trabecular VSD is very important. Brizard and colleagues ${ }^{11}$ reported that intraoperative echocardiography was useful in confirming the location of trabecular VSDs. However, their method does not identify other trabecular VSDs, which were not diagnosed preoperatively. In fact, we found multiple VSDs in 11 patients intraoperatively. Trabecular VSDs were sometimes found in the malaligned interventricular septum. In these cases, blood oozed into the right ventricular despite adequate left ventricle venting. Because missing a trabecular VSD leads to severe complications, meticulous detection is important during preoperative echocardiography and intraoperative evaluation of trabecular VSDs.

Although the felt sandwich technique can be performed without left ventriculotomy, our study showed that a large total patch area corrected in BSA for closure of VSDs might cause cardiac dysfunction, particularly in infants. Cardiac dysfunction is thought to be caused by standard VSD patch and felt patch disturbing the movement of ventricular septum. Our data suggest that postoperative cardiac function is correlated with total patch area/BSA ratio. In infants, particularly with a BSA less than $0.4 \mathrm{~m}^{2}$, cardiac function is strongly influenced by patch area. Therefore, we have made an effort to use as small a felt patch as possible. Compared with our early cases, the later cases had a felt patch that was approximately $2 \mathrm{~mm}$ smaller for VSD repair. Nevertheless, there may still be postoperative cardiac dysfunction for a large patch area. We propose PAB in cases in whom postoperative cardiac dysfunction is predicted. $\mathrm{PAB}$ can protect the pulmonary vascular bed, attenuating congestive heart failure and delaying definitive repair. $\mathrm{PAB}$, compared with primary multiple VSD repair, is considered to have the advantage of maintaining cardiac function in infants.

Intraoperative conversion to $\mathrm{PAB}$ was performed in a patient because of concern about postoperative cardiac dysfunction after placement of a large patch. The patient was a 3-month-old girl with a large solitary VSD. Her weight was $3.7 \mathrm{~kg}$ and BSA was $0.25 \mathrm{~m}^{2}$. She was scheduled for primary VSD closure, but at least 4 VSDs were detected in the trabecular septum. Postoperative cardiac function was poor (estimated ejection fraction was $31 \%$ ) presumably because of a total patch area/BSA ratio. We decided to perform $\mathrm{PAB}$ despite cardiopulmonary bypass.

\section{Conclusion}

The felt sandwich technique is simple, safe, and effective for repairing multiple trabecular VSDs. This simple technique does not require special devices, prolonged operation time, or left ventriculotomy. However, in neonates or small infants, this technique may have the risk of causing postoperative cardiac dysfunction. In patients who require large amount of patches for closure of multiple VSDs, PAB should be applied as an alternative for primary repair.

\section{References}

1. Kitagawa T, Durham LA III, Mosca RS, Bove EL. Technique and results in the management of multiple ventricular septal defects. $J$ Thorac Cardiovasc Surg. 1998;115:848-56.

2. Yamaguchi M, Yoshimura N, Oka S, Ootaki Y, Yoshida M. Closure of muscular VSD by a sandwiching method via a coexisting larger VSD or an interatrial septostomy. In: Proceedings of the 3rd World Congress of Pediatric Cardiology and Cardiac Surgery; May 27-31, 2001, Toronto, Canada:227.

3. Ootaki Y, Yamaguchi M, Yoshimura N, Oka S, Yoshida M, Hasegawa T. Surgical management of trabecular ventricular septal defects: the sandwich technique. J Thorac Cardiovasc Surg. 2003;125:508-12.

4. Serraf A, Lacour-Gayet F, Bruniaux J, Ouaknine R, Losay J, Petit J, et al. Surgical management of isolated multiple ventricular septal defects: logical approach in 130 cases. J Thorac Cardiovasc Surg. 1992;103:437-43.

5. Hanna B, Colan SD, Bridges ND, Mayer JE, Castaneda A. Clinical and myocardial status after left ventriculotomy for ventricular septal defect closure. J Am Coll Cardiol. 1991;17:110A.

6. Macé L, Dervanian P, Le Bret E, Folliguet TA, Lambert V, Losay J, et al. "Swiss cheese" septal defects: surgical closure using a single patch with intermediate fixings. Ann Thorac Surg. 1999;67:1754-9.

7. Black MD, Shukla V, Rao V, Smallhorn JF, Freedom RM. Repair of isolated multiple muscular ventricular septal defects: the septal obliteration technique. Ann Thorac Surg. 2000;70:106-10.

8. Amin Z, Gu X, Berry JM, Titus JL, Gidding SS, Rocchini AP Perventricular closure of ventricular septal defects without cardiopulmonary bypass. Ann Thorac Surg. 1999;68:149-54.

9. Okubo M, Benson LN, Nykanen D, Azakie A, Van Arsdell G, Coles $\mathrm{J}$, et al. Outcomes of intraoperative device closure of muscular ventricular septal defects. Ann Thorac Surg. 2001;72:416-23.

10. Holzer R, Balzer D, Cao QL, Lock K, Hijazi ZM. Device closure of muscular ventricular septal defects using the Amplatzer muscular ventricular septal defect occluder. J Am Coll Cardiol. 2004;43:1257-63.

11. Brizard CP, Olsson C, Willkinson JL. New approach to multiple ventricular septal defect closure with intraoperative echocardiography and double patches sandwiching the septum. J Thorac Cardiovasc Surg. 2004;128:684-92. 